\title{
IVD.2 I - Microsphere-couplings standardization with ZIKV or its E protein: The first step to construct a multiplex assay for Zika/Dengue
}

Stephanie Almeida ${ }^{1 *}$; Denise Matos ${ }^{1}$; Sheila Lima ${ }^{1}$; Alessandro Souza ${ }^{1}$; Thiago Chaves ${ }^{1}$; Marco Alberto Medeiros ${ }^{1}$; Leila Botelho ${ }^{1}$; Mariana Pierre ${ }^{1}$; Patrícia Brasil²; Adriana Azevedo ${ }^{1}$.

1Fiocruz/Bio-Manguinhos;

2Fiocruz/INI.

Introduction: Zika virus (ZIKV) was considered world emergency during Zika outbreak in Brazil in 2015. ZIKV is a clinically important arbovirus that can cause fetal neurological complications in infected pregnant women and Guillain-Barré syndrome in adults. Serological diagnosis of ZIKV infection is a challenging due to high cross-reactivity between flaviviruses, for exemple with Dengue virus (DENV). Accurate and early diagnosis is essential to properly manage the patients. Samples collected in the viremic period of the ZIKV infection are conducted for viral load measurement by molecular tests and samples collected after that period are evaluated by serological tests such as ELISA and the results are confirmed by PRNT. In the present study, we intend to construct a diagnosis test based in the liquid microarray (LIM) to detect antibodies against ZIKV and DENV simultaneously, associated to treatment with a chaotropic agent step to minimize cross-reaction.

Objective: Firstly, the objective of this work was to standardize the coupling ZIKV or its recombinant envelope protein (E-ZIKVre) on the magnetic microspheres, an important step to LIM test for Zika/Dengue.

Methodology: MagPlex ${ }^{\oplus}$ magnetic microspheres was used in purified ZIKV or E-ZIKVre couplings made in 96-well plates tested with different buffers/block solutions and evaluated by MAGPIX ${ }^{\circledast}$ device.

Results: Our data showed couplings with ZIKV and E-ZIKVre generated high values of median fluorescence intensity (MFI), when we used a specific neutral buffer associated a specific block solution. In addition, E-ZIKVre proved better than purified ZIKV particle once we detect high values of MFI and remarkable difference between patient samples tested (positive and negative for Zika serology).

Conclusion: These results suggest that coupling with E-ZIKV will contribute to a greater sensitivity and specificity for multiplex assay Zika/Dengue.

Keywords: Zika; Multiplex; Microarray 